\title{
OntoTag's Linguistic Ontologies: Improving Semantic Web Annotations for a Better Language Understanding in Machines
}

\author{
Guadalupe Aguado de Cea \\ $D L A C T, U P M$ \\ lupe@fi.upm.es
}

\author{
Asunción Gómez-Pérez \\ $D I A, U P M$ \\ asun@,fi.upm.es
}

\author{
Inmaculada Álvarez de Mon \\ DLACT, UPM \\ ialvarez@euitt.upm.es
}

Antonio Pareja-Lora
SIP,UCM/LIA,UPM
apareja@sip.ucm.es

\begin{abstract}
Following the road in-between purely linguistic annotation and solely ontology-based annotations for the Semantic Web, a hybrid (ontological and linguistic) model and platform, called OntoTag, has been created, aiming at better machine communication, interoperability and language understanding; these capabilities are derived from the incorporation into the platform of a set of linguistic ontologies, the main topic of this paper, suitable for the multi-leveled and standardized annotation of Semantic Web documents.
\end{abstract}

\section{Introduction}

Many schemas have been developed so far for the different kinds of annotation required in the field of Corpus Annotation. Besides, with the appearance of the Semantic Web [1] many other schemas, most of them based on ontologies [2, 3], have been devised for web page annotation. In this context, the semantic annotation of texts, in so far as it makes meaning explicit, has become a relevant topic; both a design of advanced models and formalisms and their application to the semantic annotation of web pages are needed.

Thus far, on the one hand, Corpus Linguistics researchers are trying to cover as many levels and aspects of annotation as possible-from a linguistic point of viewto describe language phenomena $[4,5,6,7]$; on the other hand, researchers in the Semantic Web area are focusing on:

1. Achieving a sound model of semantic annotation for web pages, that is able to capture as much knowledge from these pages as possible, so that computers can process them in a much smarter way $[8,9,10,11]$ and even be closer to understanding their meaning [12].
2. Finding the best way to describe web resources and vocabulary and the underlying meaning of web pages, mainly by means of languages like $\mathrm{XML}, \mathrm{RDF}(\mathrm{S})$ or OWL $[13,14]$.

However, there is an emerging road in-between, nowadays, that seeks to merge and sum up both kinds of annotations, combining them in order to bear a new, unified, multilingual, flexible, extensible and fully semantic model of annotation, useful for both communities $[15,16]$. Moreover, as shown by [17] "there is an increasing need for new standardization as well as urgent recognition of existing de facto standards and their transformation into International Standards". In fact, one of the main aims of the ISO - TC37SC4 committee is "to develop standards and related documents to maximize the applicability of language resources".

The OntoTag hybrid (linguistic and ontological) model for Semantic Web Annotation [16], whose linguistic ontologies we present here, is being developed following this in-between road aforementioned, as well as a number of guidelines hitherto published [18, 19, 20, 21, 22], in order to achieve the goal of standardization sought within the ISO - TC37SC4 committee, which will enable interoperability between systems, as a direct side effect, by means of sharing a common vocabulary and resource description based on ontologies.

This paper is organized as follows: firstly, some brief notes on the use of ontologies in semantic annotation will be sketched (section 2). Then, in section 3, an introduction to the state of the art in Corpus Linguistics for text annotation will be presented. In section 4, the platform OntoTag for hybrid (ontological and linguistic) annotation is shown, together with the other modules of our project, to proceed next to the discussion of OntoTag's linguistic ontologies in section 5 (some of them separately analyzed in subsections 5.1 and 5.2). Finally, some conclusions and future work will be stated-section 6-together with the 
acknowledgements section (7) and the references (section 8).

\section{Ontology-based annotations for the Semantic Web.}

As stated in [13], "ontologies have proven to be an essential element in many applications and [...] are useful not only for applications in which knowledge plays a key role, but also they can trigger a major change in current Web contents [...] leading to the third generation of the Web-known as the Semantic Web". As the same authors comment, there is much in common between the definitions of ontology and of Semantic Web given, respectively, in [23] and in [1].

From this point of view, ontologies, implemented with the newly created and standardised knowledge representation languages for the Web $[13,14]$, are called to be the ideal way to:

1. Make explicit the underlying meaning of web page terms.

2. Describe in a more machine-readable way Web resources and vocabulary.

Moreover, ontology-based annotations for the Web are expected to allow a smarter access to the resources within the WWW, to enable the inference of information from Web data and to ease the searching and browsing of web pages.

A survey on ontology based annotation tools and schemas can be found in [24].

\section{Linguistically-based annotations.}

Even though much research has been carried out by ontologists in the Semantic Web field on the semantic annotation of web pages [24], it is in the field of Corpus Linguistics where most standards, criteria and recommendations on annotation can be found. In [18], a list of the main different levels of linguistic annotation can be found, namely: lemma, morpho-syntactic, syntactic, semantic and discourse annotation. They are shown in Figure 1 (Annotation Level Pyramid), together with their corresponding tools (Linguistic Tool Stack) and applicable criteria, recommendations and guidelines (Linguistic Annotation Criteria Heap). A deep analysis of these concepts and their potential value for the Semantic Web can be found in $[15,24]$.

\section{OntoTag's hybrid annotations}

OntoTag was intended to be the platform for a standard-annotated and shared intermediate representation for the documents processed within two funded projects in

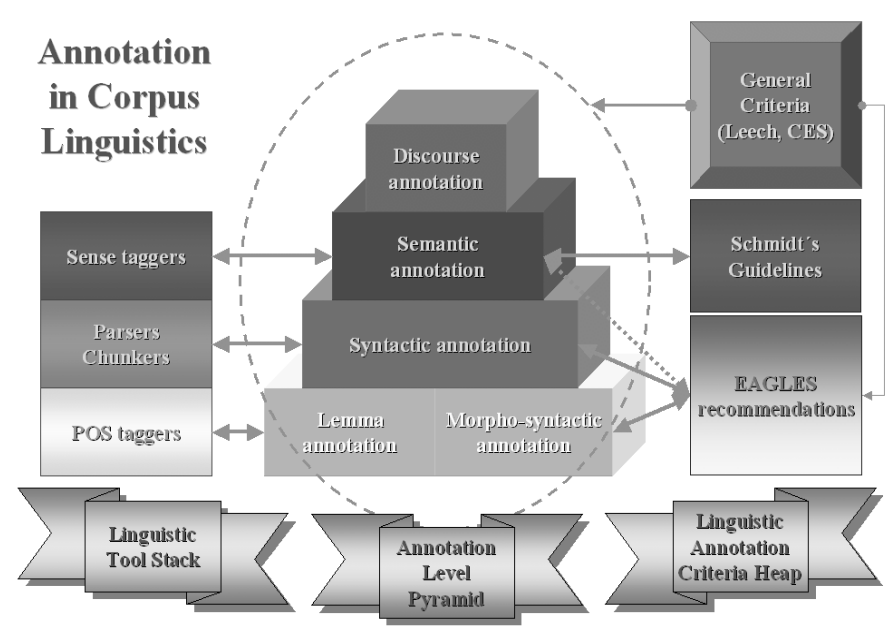

Figure 1. Linguistically based kinds of annotation.

our laboratory, ContentWeb and PLAN-H-SemWeb. As explained in [15], ontological annotations can be considered as a type of semantic annotation in the Corpus Linguistics field and, thus, OntoTag was designed as a hybrid annotation platform, that is, taking into account both ontological and linguistic annotation criteria and elements. Having in mind that, as stated by the same authors, meaning is not only within the semantic level but, instead, can be implicit and distributed among the rest of the levels, OntoTag was conceived as a multi-leveled annotation platform, in order to capture as much semantics as possible from documents.

When devising Ontotag, interoperability issues had also to be taken into consideration since it can be regarded as the base for the intercommunication and metadata storage of these other modules of ContentWeb:

1. Semi-automatic ontology learning in the domains of ecommerce and entertainment, reusing existent ontologies and international e-commerce standards and joint initiatives.

2. Development of a natural language interface based on ontologies.

3. Creation of an ontology-based system for querying and retrieving information from annotated web documents in the entertainment domain.

\section{OntoTag's linguistic ontologies}

One of the main components of the OntoTag model is its set of linguistic ontologies, devised to represent the structure and relationships between the elements of language at different linguistic levels. The kind of elements and relationships considered in these ontologies are the ones usually included in existing annotation schemas and also those already discussed in the literature 
but not implemented yet $[4,5]$ as well as some others, decided by our research team. First of all, a Linguistic Level Ontology (LLO) was developed both to capture the stratification of natural language analysis and generation and to simplify the study of the other elements. Then, following the EAGLES guidelines for morpho-syntactic annotation of corpora [18], but obviously broadening its scope, three different ontologies were built to represent the category-attribute-value formalism at all levels of annotation (morpho-syntactic, syntactic, semantic, discourse and pragmatic): a Linguistic Unit Ontology (LUO), a Linguistic Attribute Ontology (LAO), and a Linguistic Value Ontology (LVO).

Complementing these four ontologies, a fifth one (the Linguistic Pattern Ontology, LPO) has been designed for representing the patterns that these units follow when combined in an utterance. Finally, the OntoTag Integration Ontology (OIO) establishes the main relationships between documents (annotated and non-annotated), units, attributes, and values both in the linguistic and in the ontological areas of annotation.

The application of these six ontologies in the OntoTag annotation model is twofold: first, as discussed above, they identify the different elements (mostly linguistic, but also ontological) that are annotable in the Semantic Web field; second, once the ontology has been populated (instantiated) by the annotations obtained with OntoTag, they will also act as a repository or database of these annotations.
Let us now take a closer look to the four core OntoTag linguistic ontologies, namely: the LLO, the LUO, the LAO and the LVO.

\subsection{The Linguistic Level Ontology (LLO)}

As mentioned before, the Linguistic Level Ontology (LLO) was developed both to capture the stratification into different levels of natural language analysis and generation and to simplify the study of the other elements. A screen capture of this ontology is shown in Figure 2. The main sources of information for building it were the EAGLES recommendations for the morpho-syntactic and syntactic annotation of corpora $[18,19]$ and, thus the linguistic levels considered in the LUO are the morpho-syntactic, the syntactic (sub-stratified in all its layers), the semantic, the discourse, and the pragmatic levels. As commented in [19], the syntactic level was sub-divided in eight layers, namely, bracket, category, dependency relation, functional, subclassification, logical relation, rank, and non-fluency phenomena layers.

The addition of the last and upper level of Pragmatics, which was not considered in EAGLES (perhaps subsumed within the discourse level), is of a considerable interest for human communication and language understanding (and also, consequently, in human-computer interaction and communication); according to [25], Pragmatics is the study of four areas: speaker meaning (that is, what is the

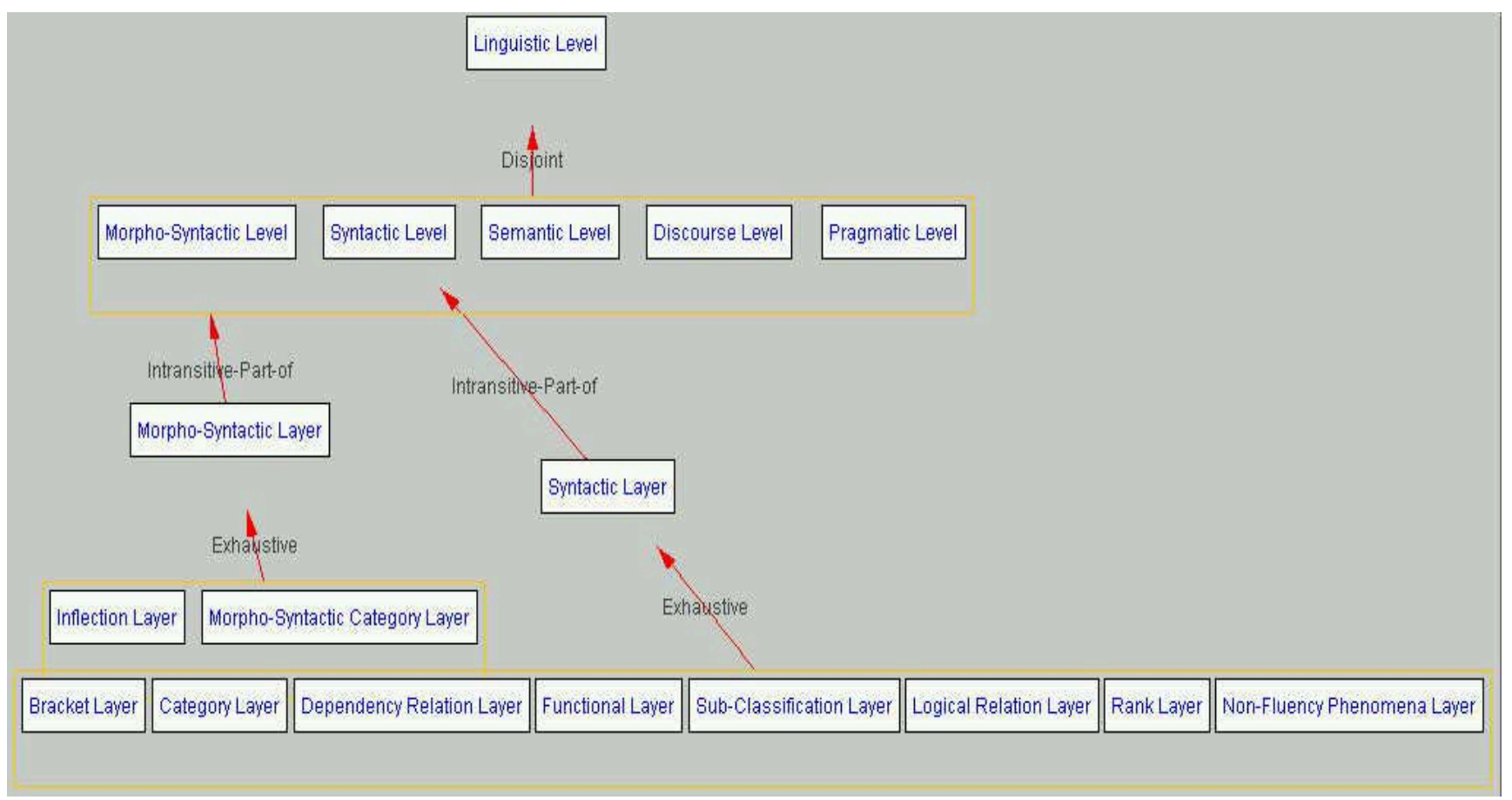

Figure 2: OntoTag's Linguistic Level Ontology (WebODE capture) 
speaker intention, independently of what he/she finally utters), contextual meaning (what people mean in a particular context and how the context influences what is said), invisible meaning (how more gets communicated than is said) and relative distance expression (how much needs to be said, according to the physical, social or conceptual subjective distance between the speaker and the listener). A description of the kind of information that the annotations at this level can give us to improve language understanding by computers can be found in the following subsection.

\subsection{The Linguistic Unit, Attribute and Value Ontologies (LUO, LAO, LVO)}

Whilst the Linguistic Unit Ontology (LUO) includes all the units (categories) identified at the different levels of annotation considered in the LLO, the Linguistic Attribute Ontology (LAO) includes the various attributes associated to the units in the LUO; the Linguistic Value Ontology (LVO) accounts for the possible values of the attributes in the LAO.

These three ontologies are presented together because of the close connection and parallelisms between them. For instance, the lemma level can be considered so simple that it has no unit associated, and, consequently, no further study for attributes or values is needed; in the morphosyntactic and in the syntactic level, the LUO incorporates all the categories included in their respective EAGLES recommendations $[18,19]$, re-structured in order to better differentiate between what can be considered an ontology class and what a class or an instance value. Therefore, a huge number of the values contained in [18] were included in the LUO as units and their associated attributes transformed in IsA (SubClassOf) relations in the LAO, since this recommendation did not differentiate two distinct layers of categorization and inflection; values such as "Common" or "Proper" (from the "Type" attribute for nouns) are included as subclasses of "Noun", the class that has associated all attributes corresponding to the morphosyntactic category of nouns.

At the semantic level, a subset of the SIMPLE ontologies [26] has been reused, after some adaptation, and attributes and values coming from Halliday's Functional Grammar [27] have been attached to these semantic units; with regard to the discourse and pragmatic levels, some units have already been identified: a reference assignment unit, that is, exophora and endophora with their subclasses, a deictic unit and its subclasses, etc. Morpho-syntactic attributes revealing a semantic, discourse or pragmatic projection have been replicated or directly assigned to the corresponding level: this is the case of "Politeness" (pragmatic) or "Person" and "Possessive number" (discourse). Named entities, included as a class of exophora at the moment, deixis and co-reference, and the aforementioned politeness or speaker-listener physical or psychological distance are the kind of elements that have been included in the model at these levels to capture as much knowledge and meaning as possible from web pages, but these and other details will require further study still.

\section{Conclusions and further work}

In this paper we have presented the set of linguistic ontologies developed for OntoTag, a Semantic Web hybrid annotation model, in order to:

a) identify the different elements (mostly linguistic, but also ontological) that are annotable in the Semantic Web field and that can make its pages more machinereadable.

b) act as a repository or database of annotations obtained with OntoTag, stored as instances in the ontologies.

The advantages of using the different linguistic ontologies presented in this paper are, on the one hand, derived from the very nature of ontologies, that is, extensibility, flexibility and, to some extent, multilinguality; the consensual nature of ontologies and the sources used in their construction $[18,19,20,21,22,25$, $26,27,28,29,30,31,32,33,34]$ also enable them (and the annotations obtained with them) to be considered standardized.

On the other hand, the meaning of a page with explicit semantic annotation obtained by means of these ontologies is reinforced by the meaning contribution provided by all of the linguistic levels; semantic analysis also benefits from the invaluable work done so far on the development of ontologies as conceptual and consensual models, which eases language understanding and communication.

However, the main disadvantage lies still in the limitations imposed by current technologies: the process of obtaining automatically annotated compact, readable, and verifiable pages is quite a hard task to be fully specified and delimited; besides, as mentioned before, further efforts must be devoted to the refinement of the discourse and the pragmatic sub-models of OntoTag and linguistic ontologies. Nevertheless, the work being done in our laboratory tries to bring some light upon these issues.

\section{Acknowledgements}

This research has been supported partly by a Ministry of Science and Technology grant (Reference TIC20012745, CONTENTWEB project) and partly by a UPM grant (Reference 14286, PLAN-H-SEMWEB project). 


\section{References}

[1] Berners-Lee, T., Fischetti, M. 1999. Weaving the Web: The Original Design and Ultimate Destiny of the World Wide Web by its Inventor. Harper. San Francisco.

[2] Gruber, T. R. 1993. "A Translation Approach to Portable Ontologies" in Journal on Knowledge Acquisition, Vol. 5(2), 199-220

[3] Borst, W. N. 1997. Construction of Engineering Ontologies. $\mathrm{PhD}$ thesis, University of Twente, Enschede.

[4] Wilson, A., Thomas, J. 1997. "Semantic Annotation". Corpus Annotation: Linguistic Information from Computer Text Corpora, R. Garside, G. Leech \& A. M. McEnery, ed., Longman, London.

[5] Schmidt, K. M. 1988. "Der Beitrag der begriffsorientierten Lexicographie zur systematischen Erfassung von Sprachwandel und das Begriffswörterbuch zur mhd. Epik". Mittelhochdeutsches Wörterbuch in der Diskussion, ed. by Bachofer, W. Tübingen: Max Niemeyer, 35-49.

[6] Pustejovsky, J., Sauri, R., Setzer, A., Gaizauskas, R., and Ingria, B. 2002. TimeML Annotation Guideline 1.00 (internal version 0.4.0). http://132.248.150.102/Volumes/esslli03/ Advanced/Pustejovsky/3TimeMLGuidelines.pdf

[7] Buitelaar, P., Declerck, T. 2003. "Linguistic Annotation for the Semantic Web". Annotation for the Semantic Web. Frontiers in Artificial Intelligence and Applications (eds.: Handschuh, S., Staab, S.). IOS Press. Amsterdam. The Netherlands.

[8] Benjamins, V.R., Fensel, D., Decker, S., Gómez-Pérez, A. 1999. "(KA $)^{2}$ : Building Ontologies for the Internet: a Mid Term Report". International Journal of Human Computer Studies (IJHCS), vol. 51. Elsevier. pp. 687-712.

[9] Motta, E., Buckingham Shum, S. Domingue, J. 1999. "Case Studies in Ontology-Driven Document Enrichment". Proceedings of the 12th Banff Knowledge Acquisition Workshop, Banff, Alberta, Canada.

[10] Luke S., Heflin J. 2000. SHOE 1.01. Proposed Specification. SHOE Project. http://www.cs.umd.edu/projects/plus/SHOE/ spec1.01.htm

[11] Handschuh, S., Staab, S. 2003. Annotation for the Semantic $W e b$. Frontiers in Artificial Intelligence and Applications. IOS Press. Amsterdam. The Netherlands.

[12] Rinaldi, F., Dowdall, J., Hess, M., Jeremy Ellman, J., Zarri, G.P., Persidis, A., Bernard, L., Karanikas, H. 2003. "Multilayer annotations in Parmenides". Proceedings of the K-CAP 2003 Workshop on Knowledge Markup \& Semantic Annotation (SemAnnot 2003 - KCap 2003 Workshop). Florida. USA.

[13] Gómez-Pérez, A.; Corcho, O. 2002. "Ontology languages for the Semantic Web". IEEE Intelligent Systems and their applications, vol. 17(1), January/February 2002. pp. 54-60.

[14] McGuinness, D.L., van Harmelen, F. 2003. OWL Web Ontology Language Overview (W3C Candidate Recommendation). August 2003. http://www.w3.org/TR/owlfeatures/

[15] Aguado, G., Álvarez-de-Mon I., Gómez-Pérez, A., ParejaLora, A., Plaza-Arteche, R. 2002. "A Semantic Web Page Linguistic Annotation Model". Semantic Web Meets Language Resources. Technical Report WS-02-16. AAAI Press. Menlo Park, California, E.E.U.U. pp. 20-29
[16] Aguado de Cea, G., Álvarez de Mon, I., Gómez-Pérez, A., Pareja-Lora, A. 2003b. "OntoTag: XML/RDF(S)/ OWL Semantic Web Page Annotation in ContentWeb". Proceedings of the 2nd Workshop on NLP and XML (NLPXML-2003) - Language Technology and the Semantic $W e b$, pp. 25-32. 10th Conference of the European Chapter of the Association for Computational Linguistics. EACL'03. Budapest, Hungary.

[17] ISO - TC37SC4. 2003. http://www.tc37sc4.org

[18] EAGLES. 1996a. EAGLES: Recommendations for the Morphosyntactic Annotation of Corpora. EAGLES Document EAG--TCWG-MAC/R.

[19] EAGLES. 1996b. EAGLES: Recommendations for the Syntactic Annotation of Corpora. EAGLES Document EAG-TCWG-SASG/1.8.

[20] XCES. 2003. Corpus Encoding Standard for XML. http://www.xml-ces.org/

[21] ISLE Computational Lexicon Working Group. 2003. Standards and Best Practice for Multilingual Computational Lexicons \& MILE (the Multilingual ISLE Lexical Entry). Deliverable D2.2-D3.2. http://www.ilc.cnr.it/EAGLES96/ isle/clwg doc/ISLE D2.2-D3.2.zip

[22] GDA. 2002. Global Document Annotation Initiative: The GDA Tag Set. http://www.i-content.org/GDA/tagset.html

[23] Studer, R., Benjamins, R., Fensel, D. 1998. "Knowledge Engineering: Principles and Methods". IEEE Transactions on Data and Knowledge Engineering, vol. 25, nos. 1-2, pp. 161-197.

[24] Aguado de Cea, G., Álvarez de Mon, I., Benjamins, R., Contreras, J., Martín, F., Navarrete, B., Pareja-Lora, A., Plaza-Arteche, R. 2003a. Esperonto Services IST-2001-34373 Deliverable D31 on Annotation Tools and Services. http://www.esperonto.net/semanticportal/esperonto/ShowDeli verables.jsp

[25] Yule, G. 1996. Pragmatics. Oxford University Press. Oxford. UK.

[26] SIMPLE Project. 2000. http://www.ub.es/gilcub/SIMPLE/ simple.html

[27] Halliday, M.A.K. 1994. An Introduction to Functional Grammar. Arnold. Bristol. UK.

[28] Dubuc, R. and Lauriston, A. 1997. "Terms and Contexts" in Wright, S.E. and G. Budin, Handbook of Terminology management Vol 1, Amsterdam/Philadelphia: John Benjamins, pp. 80-87.

[29] Faber, P. and Tercedor, M. 2000. "Codifying conceptual information in descriptive terminology management" in Meta, XLVI, 1, pp. 192-204.

[30] Mel'čuk, I. A. 1988. Dependency Syntax, New York: State University of New York Press.

[31] Mel'čuk, I. A. 1996. "Lexical functions: a tool for the description of lexical relations in a lexicon", in Wanner, L. Lexical functions in lexicography and natural language processing, John Benjamins: Amsterdam, Philadephia.

[32] Pustejovsky, J. 1998. The generative lexicon, Cambridge, Massachussets: The MIT Press.

[33] Álvarez de Mon, I. 2003. La cohesión del texto científicotécnico: un estudio contrastivo inglés-español. $\mathrm{PhD}$ thesis. Universidad Complutense de Madrid. Madrid. Spain.

[34] Brown, G., Yule, G. 1983. Discourse Analysis. Cambridge University Press. The Bath Press, Avon. UK. 